\title{
Inflammatory Pseudotumor of the Spleen: A Case Report and Literature Review
}

\author{
Andrea Ciarrocchi ${ }^{\mathrm{a}, \mathrm{c}}$, Zuleyka Bianchi ${ }^{\mathrm{a}}$, Roberto Vicentini ${ }^{\mathrm{b}}$, Massimo De Benedictis ${ }^{\mathrm{b}}$, \\ Mario Schietroma ${ }^{a}$
}

\begin{abstract}
We report a rare case of inflammatory pseudotumor (IPT) of the spleen occurred in a 28-year-old woman admitted to our hospital for left upper quadrant and epigastrium discomfort. There were no constitutional signs and laboratory findings were unremarkable. Serum oncologic markers were within ranges. A ultrasonography of the abdomen showed a solitary hypoechoic nodule within the spleen. A MRI confirmed location and morphology of the lesion. A colonoscopy and a chest X-ray were performed to rule out the presence of a primary malignancy located at other sites, but nothing relevant was found. Histological characterization with fine needle aspiration of the nodule was not performed to avoid the risk of uncontrollable bleeding and potential tumor seeding. In the suspect of a malignancy the patient underwent splenectomy. Subsequent pathologic examination of the resected organ revealed an IPT. Splenectomy resulted diagnostic and curative.
\end{abstract}

Keywords: Inflammatory pseudotumor; Benign splenic tumor; Spleen tumor

\section{Introduction}

Inflammatory pseudotumor (IPT) of the spleen is an extremely rare clinical entity and its nosology has thoroughly been revised since it was introduced in 1984 [1]. Cotelingam and Jaffe first used this term to describe a well-circumscribed splenic mass that was initially interpreted as a malignancy but then resulted histologically characterized by the predominant presence of inflammatory cells. Various classifications

\footnotetext{
Manuscript accepted for publication October 25, 2012

${ }^{a}$ Department of Surgery, General Surgery, University of L'Aquila, Italy

${ }^{\mathrm{b}}$ Department of Surgery, General and Hepatobiliopancreatic Surgery,

L'Aquila, Italy

${ }^{\mathrm{c}}$ Corresponding author: Andrea Ciarrocchi, Department of Surgery,

General Surgery, University of L'Aquila, 67100 L'Aquila, Italy.

Email: ciarro85@hotmail.it
}

doi: http://dx.doi.org/10.4021/jmc926w have been proposed in the attempt to distinguish IPTs from similar lesions with malignant potential. For this reason it is difficult to quantify the exact number of IPT reports appeared in literature. Here we specifically refer to IPT as a benign inflammatory lesion, characterized by the constant presence of inflammatory and reactive cells. With this premise to the best of our knowledge there have been less than 100 reports till now. In this paper we illustrate a case occurred in a patient presenting with nonspecific symptoms. Then we discuss the role of surgery in the management of this medical condition and its impact on long-term prognosis.

\section{Case Report}

A 28-year-old woman was admitted to hospital complaining left upper quadrant and epigastrium mild pain. Physical examination did not reveal anything significant other than a slight splenomegaly. No evidence of palpable linfoadenomegaly or masses was found. Patient's history was negative for constitutional symptoms, drug ingestion, trauma or alcohol abuse. Laboratory findings did not show anything remarkable. The patient underwent an abdomen ultrasonography (US) that documented a slight enlargement of the spleen and a rounded nodule of about 80 millimeters of diameter located in the upper pole of its parenchyma. It appeared mostly isoechoic with hypoechoic spots and undefined edges. Doppler analysis revealed peripheral vascularization. On the basis of these features it was not possible to characterize the nature of the lesion. A colonoscopy and a chest X-ray performed to rule out the presence of a primary malignancy located at other sites did not show anything abnormal. As a second level imaging technique we decided to perform a US of the abdomen with second generation echo amplifier (sulphur hexafluoride) that showed a well demarcated hypoechoic mass measuring $85 \times 63$ millimeters located in the aforementioned location with esofitic development toward the hilum (Fig. 1). It partially deformed but did not infiltrate the splenic capsule. Endovenous contrast injection showed homogeneous centripetal vascularization and subsequent progressive wash-out, which suggested an angioma. A subsequent MRI of the abdomen to further characterize the 


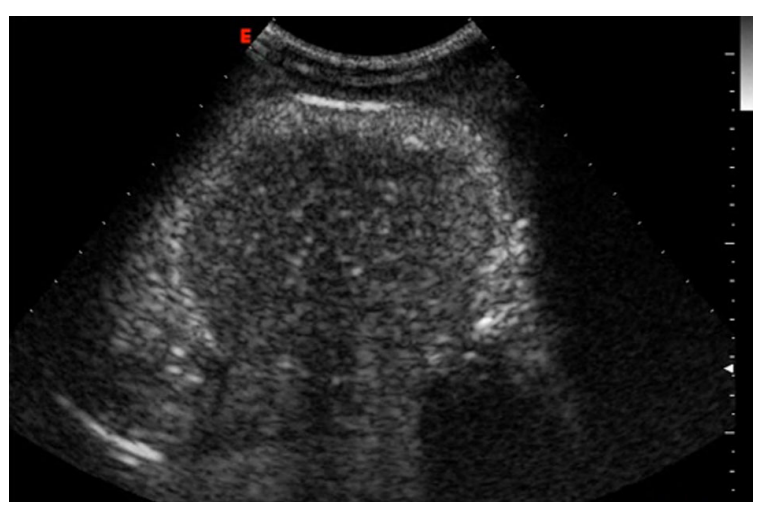

Figure 1. Hypohecoic rounded lesion appearing after echo amplifier (sulphur hexafluoride) injection.

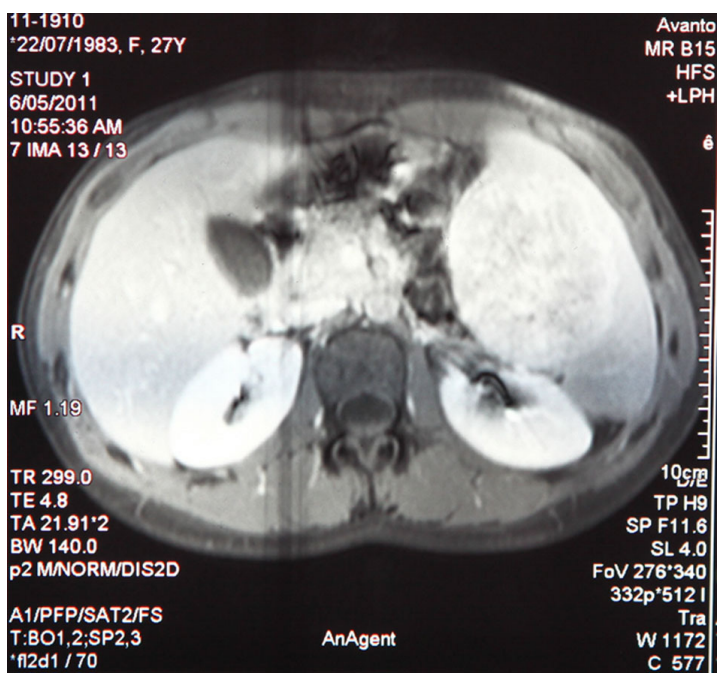

Figure 2. Isointense appearance of the nodule on T1weighted MRI.

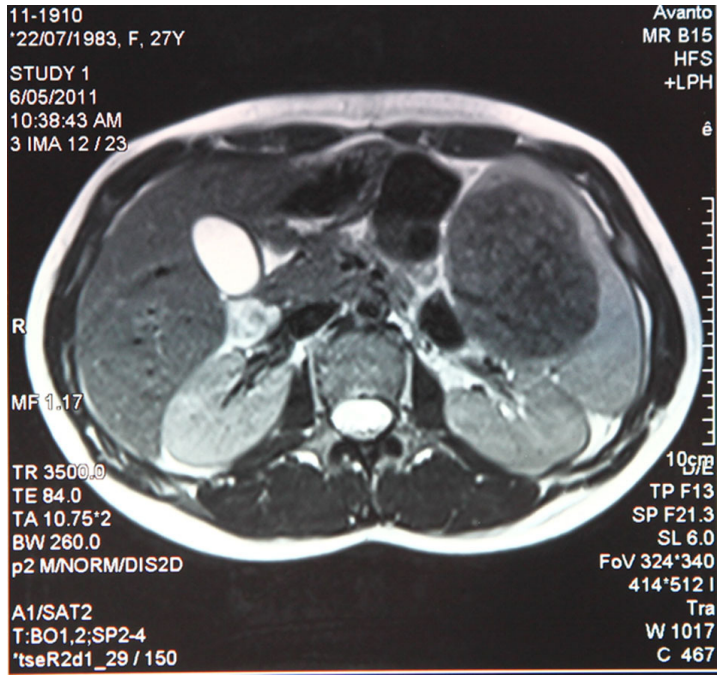

Figure 3. Markedly hypointense appearance on T2weighted MRI. mass confirmed morphology and location of the lesion, that appeared isointense on T1-weighted images and markedly hypointense on T2-weighted images with respect to the surrounding parenchyma (Fig. 2, 3). After endovenous contrast medium injection the nodule appeared dishomogeneously and diffusely vascularized. No other relevant abnormalities or linfoadenomegaly was found.

Malignancy could not be ruled out and a laparotomic splenectomy with exeresis of a lymph node of the splenic artery was successfully performed. Macroscopically the surgical specimen measured $15 \times 11.5 \times 7$ centimeters and fully contained a milky white with brownish spots nodule with rounded well-defined edges. Splenic capsule resulted integer and not infiltrated (Fig. 4). At microscopic and immunohistochemical examinations (Fig. 5) the tissue was characterized by spindle epithelioid cells, which resulted positive for vimentin and actin-smooth muscle cells and negative for desmin, cytokeratins AE1/AE3, CD34, S100, CD21, CD23 and Epstein-Barr virus (EBV) encoded RNA (EBER). CD68 resulted multifocally positive. Scattered B CD20+ lymphocytes, T CD3+ lymphocytes and numerous EMA+ or IgG4+ plasma cells were also found. The lymph node did not show pathologic abnormalities.

The patient is currently alive and asymptomatic, 15 months after surgical intervention.

\section{Discussion}

IPTs have occasionally been observed in several anatomi-

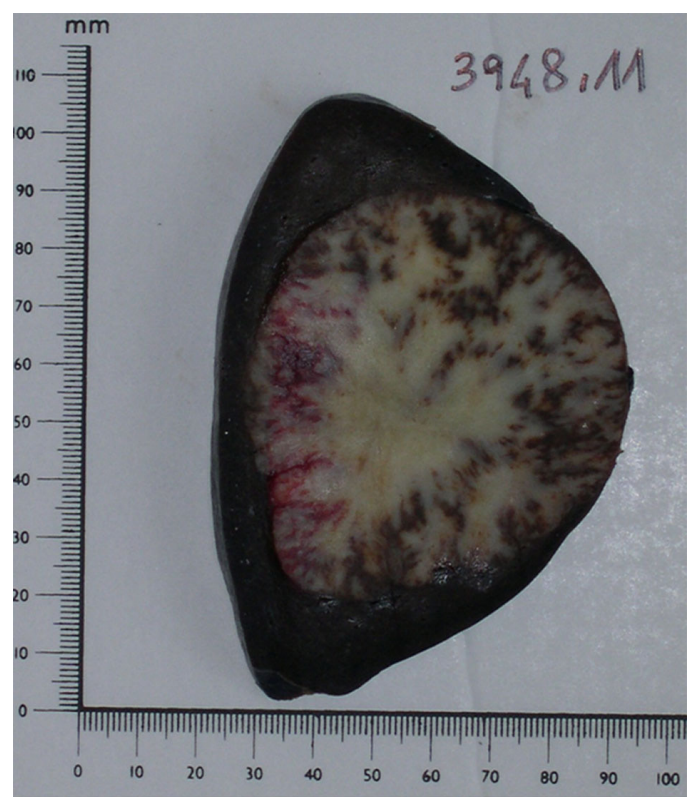

Figure 4. Surgical specimen showing a white-brownish capsulated nodule. 


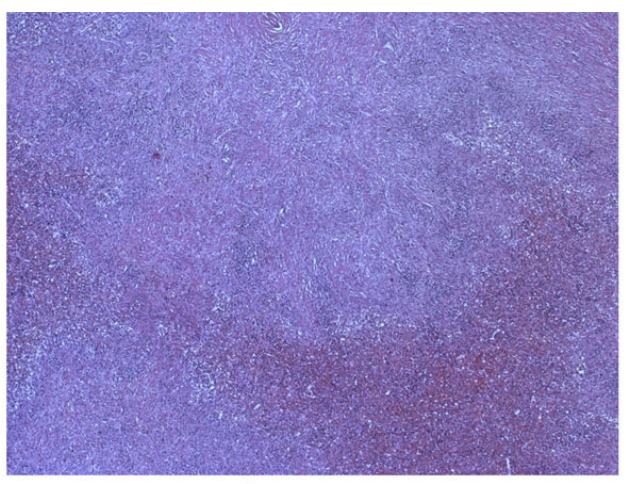

a

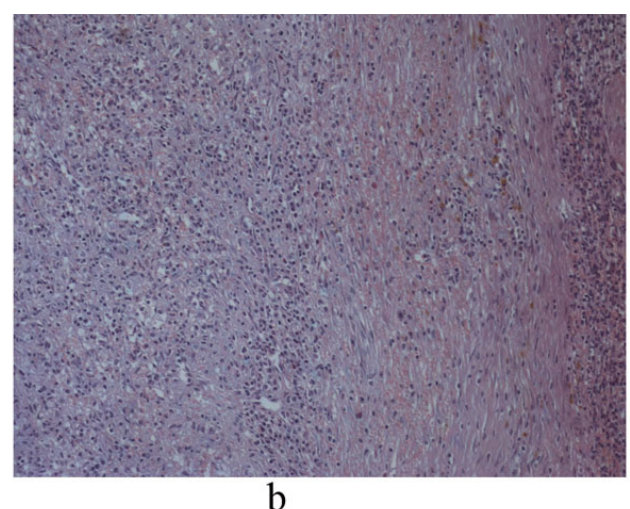

b

Figure 5. (a) Histologic section showing inflammatory and reactive cells (hematoxyline-eosin, $100 \times$ ); (b) Histologic section showing inflammatory and reactive cells (hematoxyline-eosin, $150 \times$ ).

cal locations including the respiratory tract, orbit, spinal meninges, gastrointestinal tract, soft tissues, heart and liver. Splenic involvement seems to be quite less frequent. A brief summary of epidemiologic and clinical features of reported cases was published by Moriyama S et al [2] Middle aged people of both sexes were equally affected. Diagnosis was mostly incidental or subsequent to unspecific complaints. Laboratory findings were unremarkable in most cases, however some patients presented with anemia or leukocytosis. US and CT findings appeared to be well established since the vast majority of the lesions were respectively described as hypoechoic and iso-low density nodules. On the other hand MRI patterns turned out to be more diverse, but data regarding this diagnostic technique were scarce as a result of its relatively recent introduction and limited use dictated by economic reasons. Continuous variables such as age, splenic weight and size showed ample standard deviations, reflecting population heterogeneity.

Some of the lesions originally classified as IPTs are now considered to be specific entities and some of them represent true malignancies [3] with metastatic, local invasiveness and recurrence potential. IPTs are in other words different entities each with a pathogenic mechanism sharing gross appearance on pathology and imaging. In recent years various terms and definitions have been introduced to identify each of them. Inflammatory myofibroblastic tumor is associated with ALK-1 translocation [4], IPT-like follicular dendritic cell tumor of the liver and spleen is related to clonal EBV infection [5]. Other lesions have infectious or autoimmune etiology such as mycobacterial spindle-cell IPT of lymph nodes [6] and the tumefactive lesion associated with immunoglobulin G4 (IgG4) [7]. IPTs associated to infection should be carefully evaluated to identify and treat the infectious agent in order to avoid insidious and prolonged clinical course [8]. A previous classification proposed by Someren et al [9] classified IPTs into three types: xanthogranuloma, plasma cell granuloma and sclerosing pseudotumor. Chan et al [10] proposed a classification based on the prevalent cellular type, such as infec- tive type, reparative type, myofibroblastic tumor type, follicular dendritic cell type and not otherwise specified. Both of these proposals have been abandoned since they failed to predict malignant potential, which is the determining factor in the assessment of long-term prognosis.

Etiology and pathogenesis still remain unknown but some theories have been elaborated. The initial event originally hypothesized by Cotelingam and Jaffe was necrosis and hemorrhage within the splenic parenchyma due to trauma or coagulopathy. Infectious etiology was thought because of the presence of granulomas and giant cells. Various microorganisms have been pointed out as responsible for infective IPTs such as Mycobacterium Tuberculosis [11], non tuberculous mycobacteria [12], Escherichia coli [13] gram-positive cocci [14] and Klebsiella pneumonia [15]. Detection of the infecting pathogens is obtained by bacterial cultivation and clinical response or IPT regression after administration of targeted antimicrobial agents can be considered a proof of infectious etiology. In our case inflammatory myofibroblastic tumor (IMT) [16] and EBV associated IPT-like follicular dendritic cell tumor (FOC) were excluded by immunohistochemical staining. No microorganisms were isolated from blood cultures and resected tissue. The patient did not respond as well to a serial course of treatment with antibiotics making the possibility of active infection extremely unlikely.

Another theory presumes an immune aberrant response on the basis of the high concentration of plasma cells found in many examined specimens. In our patient immunohistochemical examination showed that the lesion was positive for CD20, CD3, EMA and IgG4. Published reports of IgG4 related IPTs $[17,18]$ suggest the possibility that an autoimmune response could represent another pathogenic mechanism of IPT formation. This association has been mostly observed in patients affected by thyroiditis of Riedel or retroperitoneal idiopathic fibrosis [19].

Kawaguchi $\mathrm{T}$ et al [20] undertook in a patient with a rapidly deteriorating clinical picture steroid pulse therapy which resulted curative. The patient did not undergo surgical inter- 
vention and resulted alive and disease-free 15 months after hospital discharge. Biological rationale for steroid therapy stands on the presumed inflammatory or reactive nature of IPTs. Available data about its actual efficacy and curative potential are scarce, therefore there is still no firm indication. Further investigation should be directed to verify IPT response to steroids with comparative imaging studies. Nevertheless in the suspect of IPT in which infective etiology has been ruled out steroid administration should be taken into consideration as first line option.

Clinical presentation may be due to space occupying effect on adjacent structures or function impairment of the affected organ that in the case of the spleen can manifest as a change of blood count. Constitutional symptoms like fever, splenomegaly, sweating and fatigue can also occur. Most patients experience nonspecific symptoms such as abdominal distention, vague discomfort or mild abdominal pain. An abdominal palpable mass in the left upper quadrant is rarely reported. IPTs can also be a casual finding during diagnostic procedures performed for other reasons. Hypercalcemia [21], monoclonal peaks in the proteinogram [22] and polyclonal hypergammaglobulinemia [23] that disappear after splenectomy have also been reported.

Anatomical locations easily accessible and not in close contact with vital structures can be a target for US-guided fine needle aspiration (FNA). However we agree not to perform this procedure on the spleen, given the high risk of uncontrollable bleeding, poor specificity and theoretical potential of tumor seeding in case of malignancy [24].

The strong resemblance with malignancies on imaging techniques makes differential diagnosis difficult and often conclusive only after splenectomy. Radiolologically IPT presents as a solitary hypodense nodule. CT scans usually reveal low-density hypovascular solitary masses, sometimes with a central scar. Franquet et al [25] reported that the presence of a central stellate area corresponding to a fibrous plaque on CT examination after contrast administration is strongly suggestive of an IPT. MRI presents with isointensity on T1 weighted images and low intensity on T2 weighted images relative to the surrounding splenic parenchyma. On US IPTs usually appear as hypoechoic well-circumscribed areas [26]. Our findings were compatible with these characteristics. Differential diagnosis includes several conditions such as tumor metastases, lymphoproliferative disorders, granulomatous infections, vascular malformations, infarction, cysts, localized reactive hyperplasia and splenic hamartoma. Splenic abscesses can be easily ruled out because IPTs do not contain a fluid component. The lack of calcification and fatty elements argues against splenic hemangioma and angiosarcoma, whereas the lack of a cystic configuration argues against lymphangioma. Therefore primary splenic lymphoma and hamartoma represent the best alternatives in differential diagnosis [27].

For young children a more conservative approach has been proposed. According to Yesildag et al [28] surgery should be the option only for those cases in which tumor enlargement is apparent over time. However IPT growth over time has been observed [2], therefore size is not a reliable marker of malignancy. The great majority of papers suggest surgical treatment (open or laparoscopic) as the only available option to obtain reliable and definitive diagnosis and cure. Based on previously published reports long-term prognosis of patients who underwent splenectomy for IPT is good, except for the well-known side effects related to the loss of emunctory and immunologic functions exerted by the spleen.

\section{Conclusion}

Despite the advances of diagnostic imaging techniques in identifying space-occupying lesions, there is still lack of distinguishing features between benign and malignant masses. The increased number of USs routinely performed for any reasons will probably increase the number of IPTs detected by chance. The impossibility to predict at preoperative time the biological behavior of such masses makes splenectomy followed by specimen pathologic and immunohistochemical examinations the most secure and thus the gold standard approach to treat this condition. Steroid therapy efficacy should be further investigated.

\section{Conflict of Interest}

The authors declare no conflict of interest.

\section{References}

1. Cotelingam JD, Jaffe ES. Inflammatory pseudotumor of the spleen. Am J Surg Pathol. 1984;8(5):375-380.

2. Moriyama S, Inayoshi A, Kurano R. Inflammatory pseudotumor of the spleen: report of a case. Surg Today. 2000;30(10):942-946.

3. Lewis JT, Gaffney RL, Casey MB, Farrell MA, Morice WG, Macon WR. Inflammatory pseudotumor of the spleen associated with a clonal Epstein-Barr virus genome. Case report and review of the literature. Am J Clin Pathol. 2003;120(1):56-61.

4. Gleason BC, Hornick JL. Inflammatory myofibroblastic tumours: where are we now? J Clin Pathol. 2008;61(4):428-437.

5. Cheuk W, Chan JK, Shek TW, Chang JH, Tsou MH, Yuen NW, Ng WF, et al. Inflammatory pseudotumorlike follicular dendritic cell tumor: a distinctive lowgrade malignant intra-abdominal neoplasm with consistent Epstein-Barr virus association. Am J Surg Pathol. 2001;25(6):721-731. 
6. Kutok JL, Pinkus GS, Dorfman DM, Fletcher CD. Inflammatory pseudotumor of lymph node and spleen: an entity biologically distinct from inflammatory myofibroblastic tumor. Hum Pathol. 2001;32(12):1382-1387.

7. Yamamoto H, Yamaguchi H, Aishima S, Oda Y, Kohashi K, Oshiro Y, Tsuneyoshi M. Inflammatory myofibroblastic tumor versus IgG4-related sclerosing disease and inflammatory pseudotumor: a comparative clinicopathologic study. Am J Surg Pathol. 2009;33(9):1330-1340.

8. Lee SH, Fang YC, Luo JP, Kuo HI, Chen HC. Inflammatory pseudotumour associated with chronic persistent Eikenella corrodens infection: a case report and brief review. J Clin Pathol. 2003;56(11):868-870.

9. Someren A. "Inflammatory pseudotumor" of liver with occlusive phlebitis: report of a case in a child and review of the literature. Am J Clin Pathol. 1978;69(2):176-181.

10. Chan JKC. Inflammatory pseudotumor: a family of lesions of diverse nature and etiologies. Am J Surg Pathol 1995; 19:859-872.

11. Sekosan M, Cleto M, Senseng C, Farolan M, Sekosan J. Spindle cell pseudotumors in the lungs due to Mycobacterium tuberculosis in a transplant patient. Am J Surg Pathol. 1994;18(10):1065-1068.

12. Suster S, Moran CA, Blanco M. Mycobacterial spindle-cell pseudotumor of the spleen. Am J Clin Pathol. 1994;101(4):539-542.

13. Standiford SB, Sobel H, Dasmahapatra KS. Inflammatory pseudotumor of the liver. J Surg Oncol. 1989;40(4):283-287.

14. Lupovitch A, Chen R, Mishra S. Inflammatory pseudotumor of the liver. Report of the fine needle aspiration cytologic findings in a case initially misdiagnosed as malignant. Acta Cytol. 1989;33(2):259-262.

15. Kafeel G, Telesinghe PU. Inflammatory pseudotumour of the liver. J Clin Pathol. 1997;50(4):352-353.

16. Cessna MH, Zhou H, Sanger WG, Perkins SL, Tripp S, Pickering D, Daines C, et al. Expression of ALK1 and p80 in inflammatory myofibroblastic tumor and its mesenchymal mimics: a study of 135 cases. Mod Pathol. 2002;15(9):931-938.

17. Zen Y, Harada K, Sasaki M, Sato Y, Tsuneyama K, Haratake J, Kurumaya H, et al. IgG4-related sclerosing cholangitis with and without hepatic inflammatory pseudotumor, and sclerosing pancreatitis-associated sclerosing cholangitis: do they belong to a spectrum of scle- rosing pancreatitis? Am J Surg Pathol. 2004;28(9):11931203.

18. Noguchi H, Kondo H, Kondo M, Shiraiwa M, Monobe Y. Inflammatory pseudotumor of the spleen: a case report. Jpn J Clin Oncol. 2000;30(4):196-203.

19. Krishnan J, Frizzera G. Two splenic lesions in need of clarification: hamartoma and inflammatory pseudotumor. Semin Diagn Pathol. 2003;20(2):94-104.

20. Kawaguchi T, Mochizuki K, Kizu T, Miyazaki M, Yakushijin T, Tsutsui S, Morii E, et al. Inflammatory pseudotumor of the liver and spleen diagnosed by percutaneous needle biopsy. World J Gastroenterol. 2012;18(1):90-95.

21. Shepherd JJ, Fassett RG, Challis D. Hypercalcaemia associated with inflammatory pseudotumour of the spleen. Br J Surg. 1992;79(1):50.

22. Galindo Gallego M, Ortega Serrano MP, Ortega Lopez M, Esteban Collazo F, Guinea Esquerdo L. Inflammatory pseudotumor of spleen. Report of two cases and literature review. Minerva Chir. 1997;52(11):1379-1388.

23. Dalal BI, Greenberg H, Quinonez GE, Gough JC. Inflammatory pseudotumor of the spleen. Morphological, radiological, immunophenotypic, and ultrastructural features. Arch Pathol Lab Med. 1991;115(10):10621064.

24. Takamoto K, Midorikawa Y, Minagawa M, Makuuchi M. Inflammatory pseudotumor of the spleen: clinical impact in surgical treatment. Biosci Trends. 2007;1(2):113116.

25. Franquet T, Montes M, Aizcorbe M, Barberena J, Ruiz De Azua Y, Cobo F. Inflammatory pseudotumor of the spleen: ultrasound and computed tomographic findings. Gastrointest Radiol. 1989;14(2):181-183.

26. Ma PC, Hsieh SC, Chien JC, Lao WT, Chan WP. Inflammatory pseudotumor of the spleen: CT and MRI findings. Int Surg. 2007;92(2):119-122.

27. Suga K, Miura K, Kume N, Hara A, Matsunaga N, Tangoku A, Oka M. Tc-99m colloid and Ga-67 imaging of splenic inflammatory pseudotumor correlation with ultrasound, CT, and MRI. Clin Nucl Med. 1999;24(5):334337.

28. Yesildag E, Sarimurat N, Ince U, Numan F, Buyukunal C. Nonsurgical diagnosis and management of an inflammatory pseudotumor of the spleen in a child. J Clin Ultrasound. 2003;31(6):335-338. 